\title{
Hipertensão pulmonar em pneumopatias crônicas: temos que aprender mais
}

\author{
Pulmonary hypertension in chronic respiratory disorders: we need to learn more
}

\author{
Roberto F. P. Machado
}

\begin{abstract}
A hipertensão pulmonar é uma complicação freqüente das pneumopatias crônicas, tais como doença pulmonar obstrutiva crônica, fibrose pulmonar idiopática e apnéia do sono. Estima-se que a prevalência da hipertensão pulmonar em pacientes hospitalizados em decorrência de doenças respiratórias seja de 28\%.(1) Em pacientes com doença pulmonar obstrutiva crônica (DPOC) ou fibrose pulmonar idiopática
\end{abstract} referidos para transplante pulmonar ou cirurgia de redução de volume pulmonar, a prevalência da hipertensão pulmonar - definida como pressão arterial pulmonar média (PAPm) $>25 \mathrm{mmHg}$ - também se aproxima de 30\%. ${ }^{(2-4)}$ Em geral, a severidade da hipertensão pulmonar nestes pacientes tende a ser leve, com PAPm entre 25-35 mmHg. Na maioria dos casos, a severidade da hipertensão pulmonar está diretamente relacionada com a severidade da doença pulmonar e 0 grau de hipoxemia. Contudo, a correlação não é forte, e em alguns casos, pacientes apresentam hipertensão pulmonar severa que é desproporcional à severidade da doença pulmonar. Por exemplo, Thabut et al. identificaram 16 entre 215 pacientes com DPOC avançada e hipertensão pulmonar severa (PAPm 39,8 \pm 10,2 mmHg) e, em contraste, diminuição mais moderada no volume expiratório forçado no primeiro segundo (VEF $; 48,5 \pm 11,8 \%$ do predito). ${ }^{(5)}$ Em um grupo de 28 pacientes com fibrose pulmonar idiopática, Leuchte et al. identificaram 6 pacientes com PAPm $>35$ mmHg. ${ }^{(6)}$

Parece claro que a vasoconstrição hipóxica crônica não é o único mecanismo associado à patogênese da hipertensão pulmonar em pacientes com pneumopatias crônicas. Indivíduos vivendo em altitude desenvolvem hipertensão pulmonar e hipertrofia da camada média da artéria pulmonar, que são reversíveis com o retorno ao nível do mar. Além disso, o uso crônico de oxigênio não reverte a hipertensão pulmonar em pacientes com DPOC ou fibrose pulmonar, o que sugere que existe um remodelamento da circulação arterial pulmonar. Em contraste com indivíduos vivendo em altitude, em pacientes com DPOC ou fibrose pulmonar idiopática e hipertensão pulmonar, todas as camadas da parede da arterial pulmonar são afetadas. ${ }^{(7)}$ Estas mudanças são mais proeminentes na camada íntima, que se espessa pela presença de miócitos e pelo depósito de colágeno e elastina no espaço extracelular. Além disso, em pacientes com fibrose pulmonar idiopática, as lesões da camada íntima podem progredir para fibrose acelular e obstrução do lúmen arterial, o que é mais proeminente no foco fibroblástico, uma área que apresenta densidade vascular extremamente baixa. ${ }^{(8)}$ Os mecanismos biológicos envolvidos nestas alterações histológicas não estão bem definidos, mas parecem envolver anormalidades nos mesmos mediadores implicados na patogênese da hipertensão arterial pulmonar idiopática, tais como óxido nítrico, endotelina e prostaciclinas.

A presença de hipertensão pulmonar tem um impacto deletério na sobrevida de pacientes com pneumopatias crônicas. Em uma coorte de pacientes com DPOC, a sobrevida em 5 anos foi de $36 \%$ nos indivíduos com hipertensão pulmonar e de $62 \%$ nos pacientes sem hipertensão pulmonar. ${ }^{(9)}$ Em casos de hipertensão pulmonar severa, a sobrevida média é de 26 meses. ${ }^{(2)}$ Em pacientes com fibrose pulmonar idiopática com pressão arterial pulmonar sistólica estimada por ecocardiograma > $50 \mathrm{mmHg}$, a sobrevida média é de 0,7 anos; a sobrevida é superior a 4 anos naqueles com pressão arterial pulmonar sistólica $<50 \mathrm{mmHg} .{ }^{(10)}$ Esses achados foram confirmados em pacientes com PAPm > $25 \mathrm{mmHg}$ : em pacientes com hipertensão pulmonar, a taxa de mortalidade em 1 ano foi de 28\% contra 5,5\% naqueles sem hipertensão pulmonar. ${ }^{(3)}$ Leuchte et al. avaliaram fatores prognósticos em 176 pacientes com doenças pulmonares crônicas, incluindo, entre outras, DPOC, fibrose pulmonar, fibrose cística, sarcoidose e bronquiectasias. ${ }^{(11)}$ Neste estudo, elevações nos níveis do peptídeo natriurético cerebral, $\mathrm{PAPm} \geq 35 \mathrm{mmHg}$, resistência vascular pulmonar $\geq 320$ dina.s. $\mathrm{cm}^{-5}$ e débito cardíaco $\leq 4.4 \mathrm{l} / \mathrm{min}$ foram associados com o aumento da mortalidade, independentemente de anormalidades em provas de função pulmonar. Dentro desse contexto, o estudo de Rovedder et al. publicado nessa edição do Jornal Brasileiro de Pneumologia nos traz mais evidências de que a hipertensão pulmonar ocorre freqüentemente em pacientes com fibrose cística. ${ }^{(12)}$ Os autores avaliaram a presença da hipertensão pulmonar por meio de ecocardiograma em 37 pacientes com fibrose cística, usando a velocidade de regurgitação tricúspide (VRT) para estimar a pressão arterial pulmonar sistólica. Usando pontos de corte para o VRT de $2,5 \mathrm{~m} / \mathrm{s}$ e $2,8 \mathrm{~m} / \mathrm{s}$, a prevalência de hipertensão pulmonar foi de $49 \%$ e $30 \%$, respectivamente. Nos pacientes com hipertensão pulmonar, os valores de $\mathrm{SpO}_{2}$ em repouso e após exercício, capacidade vital forçada e $\mathrm{VEF}_{1}$ foram significativamente menores, e a VRT correlacionou-se com vários marcadores de severidade da doença pulmonar. Esses importantes achados confirmam os resultados de outros estudos nesta população e sugerem 
que a hipertensão pulmonar está ligada à severidade da doença pulmonar. Infelizmente o estudo não aborda as conseqüências funcionais da hipertensão pulmonar nestes pacientes. Por exemplo, seria interessante investigar, em pacientes com função pulmonar semelhante, se a hipertensão pulmonar resulta em diminuição na distância percorrida no teste de caminhada de seis minutos.

Tendo em vista a alta prevalência de certas pneumopatias crônicas como a DPOC, as doenças pulmonares estão certamente entre as causas mais freqüentes de hipertensão pulmonar em geral. Além disso, a presença da hipertensão pulmonar é no mínimo um marcador, e muito provavelmente uma causa, de pior prognóstico neste grupo de pacientes. Apesar disso, sabemos muito pouco sobre o manejo destes pacientes. Além do tratamento da doença de base, do uso de oxigênio e do transplante pulmonar, não existem dados robustos sobre o tratamento específico da hipertensão pulmonar. Estudos não controlados, com um pequeno número de pacientes, utilizando prostaciclinas, bosentana, sildenafil e óxido nítrico inalatório sugerem que estes fármacos podem ter um efeito benéfico e não atenuam a vasoconstrição pulmonar hipóxica, o que poderia resultar em piora na oxigenação sistêmica. Por exemplo, o uso agudo de sildenafil em pacientes com fibrose pulmonar e PAPm $>35 \mathrm{mmHg}$ resultou em melhora do quadro hemodinâmico sem efeitos adversos sobre a oxigenação. ${ }^{(13)}$ Em duas séries de casos com pacientes com DPOC e fibrose pulmonar idiopática, o uso de sildenafil por 8 a 12 semanas resultou em melhora hemodinâmica e da capacidade funcional. ${ }^{(14,15)}$ Esperamos que estes resultados preliminares gerem suficiente entusiasmo para a realização de estudos randomizados e placebo-controlados nesta população. Por enquanto não se pode recomendar com segurança o tratamento da hipertensão pulmonar em pacientes com pneumopatias crônicas. Contudo, na opinião do autor, deve-se considerar de forma individualizada o tratamento de pacientes sintomáticos e com elevações moderadas a severas da PAPm, especialmente naqueles em que a severidade da hipertensão pulmonar é desproporcionalmente maior do que a severidade da doença pulmonar de base.

Roberto F. P. Machado

Pulmonary and Vascular Medicine Branch, National Heart Lung and Blood Institute, National Institutes of Health

\section{Referências}

1. Hyduk A, Croft JB, Ayala C, Zheng K, Zheng ZJ, Mensah GA. Pulmonary hypertension surveillance--United States, 19802002. MMWR Surveill Summ. 2005;54(5):1-28.

2. Chaouat A, Bugnet AS, Kadaoui N, Schott R, Enache 1, Ducoloné A, et al. Severe pulmonary hypertension and chronic obstructive pulmonary disease. Am J Respir Crit Care Med. 2005;172(2):189-94.

3. Lettieri CJ, Nathan SD, Barnett SD, Ahmad S, Shorr AF. Prevalence and outcomes of pulmonary arterial hypertension in advanced idiopathic pulmonary fibrosis. Chest. 2006;129(3):746-52.

4. Scharf SM, lqbal M, Keller C, Criner G, Lee S, Fessler HE. Hemodynamic characterization of patients with severe emphysema. Am J Respir Crit Care Med. 2002;166(3):314-22.

5. Thabut G, Dauriat G, Stern JB, Logeart D, Lévy A, MarrashChahla R, et al. Pulmonary hemodynamics in advanced COPD candidates for lung volume reduction surgery or lung transplantation. Chest. 2005;127(5):1531-6.

6. Leuchte HH, Neurohr C, Baumgartner R, Holzapfel M, Giehrl $\mathrm{W}$, Vogeser $\mathrm{M}$, et al. Brain natriuretic peptide and exercise capacity in lung fibrosis and pulmonary hypertension. Am J Respir Crit Care Med. 2004;170(4):360-5.

7. Naeije R, Barbera JA. Pulmonary hypertension associated with COPD. Crit Care. 2001;5(6):286-9.

8. Ebina M, Shimizukawa M, Shibata N, Kimura Y, Suzuki T, Endo $\mathrm{M}$, et al. Heterogeneous increase in CD34-positive alveolar capillaries in idiopathic pulmonary fibrosis. Am J Respir Crit Care Med. 2004;169(11):1203-8.

9. Oswald-Mammosser M, Weitzenblum E, Quoix E, Moser G, Chaouat A, Charpentier C, et al. Prognostic factors in COPD patients receiving long-term oxygen therapy. Importance of pulmonary artery pressure. Chest. 1995;107(5):1193-8.

10. Nadrous HF, Pellikka PA, Krowka MJ, Swanson KL, Chaowalit $\mathrm{N}$, Decker PA, et al. The impact of pulmonary hypertension on survival in patients with idiopathic pulmonary fibrosis. Chest. 2005;128(6 Suppl):616S-7S.

11. Leuchte HH, Holzapfel M, Baumgartner RA, Ding l, Neurohr C, Vogeser M, et al. Clinical significance of brain natriuretic peptide in primary pulmonary hypertension. J Am Coll Cardiol. 2004;43(5):764-70.

12. Rovedder PM, Ziegler B, Pinotti AF, Barreto SS, Dalcin, PT. Prevalence of pulmonary hypertension evaluated by Doppler echocardiography in a population of adolescent and adult patients with cystic fibrosis. J Bras Pneumol. 2008;34(2):83-90.

13. Ghofrani HA, Wiedemann R, Rose F, Schermuly RT, Olschewski H, Weissmann N, et al. Sildenafil for treatment of lung fibrosis and pulmonary hypertension: a randomised controlled trial. Lancet. 2002;360(9337):895-900.

14. Alp S, Skrygan M, Schmidt WE, Bastian A. Sildenafil improves hemodynamic parameters in COPD--an investigation of six patients. Pulm Pharmacol Ther. 2006;19(6):386-90.

15. Madden BP, Allenby M, Loke TK, Sheth A. A potential role for sildenafil in the management of pulmonary hypertension in patients with parenchymal lung disease. Vascul Pharmacol. 2006;44(5):372-6. 